\title{
Small Teeth Difference Transmission Interference Theory Analysis
}

\author{
Jia Ming Feng
}

School of Mechanical Engineering, JILIN Agricultural Science and Technology College, JiLin132101 China;

491327847@qq.com

\section{Keywords: Interference Analysis, small teeth difference, transmission.}

\begin{abstract}
Small teeth difference planetary gear is a planetary gear drive, the drive ring plate is a special form of few teeth difference planetary transmission, by double crank gear mechanism guiding circular translational research object of this paper is a bicycles ring transmission part of the gear condition interference does not occur. On the basis of previous error factors it takes into account the actual tooth profile overlap interference limits the minimum allowable value calculation formula, where in the center distance error and the impact of the introduction of tooth thickness deviation tooth profile interference, simultaneous analysis of both its impact sensitivity. A reasonable allocation of parts machining tolerances, tooth profile to avoid interference, lower manufacturing costs provide a theoretical basis.
\end{abstract}

\section{Introduction}

The small teeth is a kind of planetary gear transmission, which is composed of an external gear and an internal gear to form a pair of internal gear pair, the difference between the number of teeth inside and outside the gear less, so referred to as the number of teeth less differential drive. The small of teeth is usually 1 tooth, 2 teeth, 3 teeth, etc. Ring plate drive is a special form of small number of teeth difference planetary transmission ${ }^{[1]}$, through the double crank gear mechanism for circular translation. According to parallel quadrilateral, the ring-plate drive can be divided into two rings, tricycles, four-ring and other forms and the object of this study for the double-loop transmission ring part of the transmission. This article study the condition of the internal contact does not interfere. The influence of some practical factors on the internal meshing interference is considered, which provides the theoretical reference for the design of the double - loop transmission and provides the technical reference for the manufacturing assembly. Figure 1 for the double-loop transmission mechanism diagram.

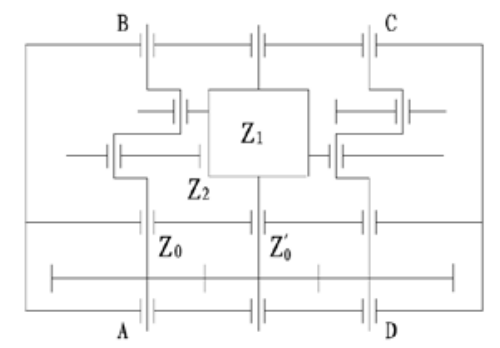

Fig. 1 Diagram of double-loop transmission.

In the internal meshing drive, it is possible that the tooth profile that does not overlap at the meshing position is overlapped with each other. This phenomenon is called tooth profile overlap interference, or tooth tip angle interference. In many literatures, it has been given that the constraint condition of internal interference is less than $0^{[2]}$, that is, the theoretical condition of noninterference, but the derivation of this condition does not take into account the influence of practical factors on overlapping interference such as machining error and Installation error. The influence of these actual error pairs is described in literature ${ }^{[3]}$. On the basis of this literature, the influence of center distance error and tooth thickness deviation is added. This article studies tooth profile interference condition base on these kinds of errors. 


\section{The effect of center distance error on [Gs]}

The relationship between the center distance of the gear pair in the inner meshing and the meshing angle is:

$$
\cos \alpha^{\prime}=a \cdot \cos \alpha / a^{\prime}
$$

Which $a$ is the standard center distance; ${ }^{a^{\prime}}$ for the design center distance. From the formula 1 can be further obtained $a^{\prime}{ }_{\text {with }} \alpha_{a}{ }^{\prime}$ the relationship between:

$$
\alpha_{a}^{\prime}=\arccos \left(\frac{a \cdot \cos \alpha}{a^{\prime}+\Delta a}\right)
$$

Which $\Delta a$ is the center distance error; ${ }^{\alpha}{ }^{\prime}$ for the gear center from the center of the error after the actual engagement angle.

Under the premise of considering only the deviation of the center, through the formula $\left[G_{S}\right]=\left(z_{2}-z_{1}\right) \cdot\left(\tan \alpha^{\prime}-\tan \alpha_{a}{ }^{\prime}+\Delta \alpha_{a}\right)+z_{1} \gamma_{1}+z_{2} \gamma_{2}$, we can draw the formula ${ }^{\left[G_{s}\right]}$ :

$$
\left[G_{S}\right]=\left(z_{2}-z_{1}\right) \cdot\left(\tan \alpha^{\prime}-\tan \alpha_{a}{ }^{\prime}+\Delta \alpha_{a}\right)
$$

Which is $\alpha_{a}^{\prime}=\arccos \left(\frac{a \cdot \cos \alpha}{a^{\prime}+\Delta a}\right), \Delta \alpha_{a}=\alpha_{a}{ }^{\prime}-\alpha^{\prime}$ 。

There are two main factors influencing the center distance error, namely, the deviation of the crank center error, that is, the center distance deviation $f_{a}$; the radial run out error of the ring gear $F_{r}$, the error is mainly caused by the geometric eccentricity of the gear $e_{r} . e_{r}=F_{r} / 2$, Due to the existence of $e_{r}$, when the gear meshing, the actual center distance from the theoretical center distance on the basis of increased ${ }^{ \pm 2 e_{r}}$, s fluctuations [4]. This paper only studies the effect of the error on the interference generated by the center from the maximum eccentricity $\Delta a=F_{r}+f_{a}$. In this paper, the ring-type transmission Fr is $0.041 \mathrm{~mm}$, crank eccentricity deviation of $\pm 0.05 \mathrm{~mm}$, so $\Delta a= \pm 0.091 \mathrm{~mm}$. Fig. 2 shows the effect of the center distance error pair ${ }^{\left[G_{s}\right]}$. From Fig. 2, it can be concluded that there is no other error in the case where the center distance error is negative, then the corresponding value is positive. The effect of the center pair error pair is that it decreases with the increase of the center distance error, so the center distance deviation should be as non-negative as possible, which can reduce the occurrence of the interference phenomenon.

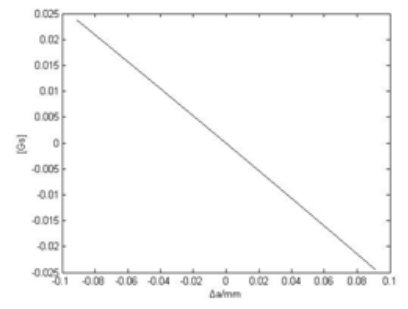

Fig. $2 \Delta a$ on the $[\mathrm{Gs}]$ impact

\section{Effect of tooth thickness deviation on [Gs]}

Tooth thickness deviation refers to the sub-cylindrical surface, the thickness of the century and the nominal tooth thickness difference. Tooth thickness on the deviation of Esns, is the minimum thickness of the tooth thickness; tooth thickness deviation Esni is the maximum thickness of the amount of thinning. By the formula $\left[G_{S}\right]=\left(z_{2}-z_{1}\right) \cdot\left(\tan \alpha^{\prime}-\tan \alpha_{a}{ }^{\prime}+\Delta \alpha_{a}\right)+z_{1} \gamma_{1}+z_{2} \gamma_{2}$ can be gotten [5], only consider the conditions of tooth thickness deviation, there is no other error in the case,

$$
\left[G_{s}\right]=z_{1} \gamma_{1}+z_{2} \gamma_{2}
$$


Which is,

$$
\gamma_{1}=-\gamma_{E s 1}=-\frac{\left|E_{s 1}\right|}{r_{1}}=-\frac{2\left|E_{s 1}\right|}{m z_{1}} \quad \gamma_{2}=-\gamma_{E s 2}=-\frac{\left|E_{s 2}\right|}{r_{2}}=-\frac{2\left|E_{s 2}\right|}{m z_{2}}
$$

$\left|E_{S 1}\right|+\left|E_{S 2}\right|=E_{S}$ Which z1, z2 and r1, r2 are the number of teeth outside the internal gear and the radius of the index circle. Then, we can get it:

$$
\left[G_{s}\right]=-\frac{2}{m}\left(\left|E_{S 1}\right|+\left|E_{S 2}\right|\right)=-\frac{2}{m} E_{S}
$$

In this paper, the gear accuracy of the object is 7 , the upper and lower deviation of the tooth thickness is $\mathrm{HK}$ and the pitch deviation is $f_{p t}=20 \mu \mathrm{m}$. The upper and lower deviations of the tooth thickness are:

$$
\left\{\begin{array}{l}
E_{s n s}=-8 f_{p t}=-8 \times 20 \mu \mathrm{m}=-160 \mu \mathrm{m}=-0.016 \mathrm{~mm} \\
E_{s n i}=-12 f_{p t}=-12 \times 20 \mu \mathrm{m}=-240 \mu \mathrm{m}=-0.024 \mathrm{~mm}
\end{array}\right.
$$

Tolerance of tooth thickness is $\left|E_{s 1}\right|=\left|E_{s 2}\right|=-E_{s n i}-E_{s n s}=0.04 \mathrm{~mm}$

The influence of the upper and lower deviation pairs of the internal gear tooth thickness is shown in Fig3. It can be seen from the figure, the internal and external gear tooth thickness deviation on the degree of impact is the same. By the tooth thickness deviation of the impact ${ }^{\left[G_{s}\right]}$ of non-positive; with the tooth thickness deviation decreases, also $\left[G_{s}\right]$ reduced, the more likely to cause interference.

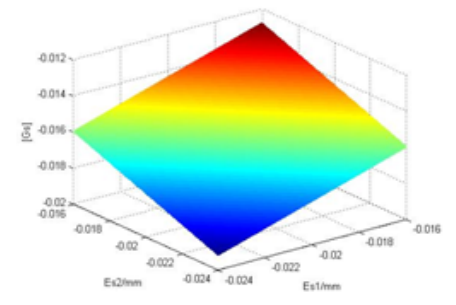

Figure 3 Effects of ES1 and ES2 on [Gs].

\section{Two kinds of errors on the interference sensitivity analysis}

In this paper, we consider only the influence of single error pair ${ }^{\left[G_{s}\right]}$, and we will study the interference sensitivity of center distance error and tooth thickness error. From the formula (3) and (5) can be obtained from the center error and tooth thickness error on the comparison chart, as shown in Figure 4. It can be seen from Fig. 4 that the effect ${ }^{\left[G_{s}\right]}$ of the tooth thickness error pair is much greater than the influence of the center distance error pair. At the same time, it can be concluded that the tooth thickness deviation can be reduced ${ }^{\left[G_{s}\right]}$, because the tooth thickness deviation of the existence of tooth profile interference phenomenon is not easy to occur. It can be seen from Fig. 4 that the sensitivity of tooth thickness deviation to tooth profile interference is much higher than that of center distance error.

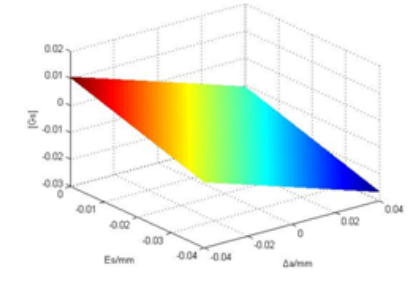

Fig. 4 Effect of $\Delta \mathrm{a}$ and $\mathrm{ES}$ on $[\mathrm{Gs}]$ 


\section{Summary}

Based on the study of predecessors, this paper continues to study the ${ }^{\left[G_{s}\right]}$ calculation of the allowable value of the tooth profile overlap interference constraint condition considering the actual error factors, which introduces the influence of the center distance error and the tooth thickness deviation on the tooth profile interference. The influence of the distance error and the tooth thickness deviation on the tooth profile interference is analyzed, and the sensitivity of the tooth profile interference parameters to different errors is determined, and the processing of the parts is also distributed rationally tolerance. Avoiding tooth profile interference, reduce manufacturing costs to provide theoretical basis.

\section{ACKNOWLEDGEMENTS}

Fund Project: Jilin Agricultural Science and Technology Institute Youth Fund Project [2016] No. Q18

\section{References}

[1] Zhou Youqiang, Hu Mao-hyun, Zhang Wen-zhao. Development of small tooth difference transmission [J]. Gear, 1983 (1).

[2] Zhu Xiaobo. Analysis and Checking of Interference in Internal Gear [J]. Journal of Yancheng Institute of Technology .1995.8 (4).

[3] Cheng Da first editor. Mechanical design manual (fourth edition) Volume 2. Beijing: Chemical Industry Press editor .2004.1.

[4] YIN Hai-yan, YANG Xi-he.K.H type three-ring reducer research [J]. Mechanical transmission .1992,16 (4).

[5] Dong Zhen. Dual-loop transmission interference and dynamic simulation analysis [D]. Northeastern University, 2012.

[6] Li Yuan. Kinematic simulation and load carrying capacity calculation of three-ring reducer [An]: Anshan: Liaoning University of Science and Technology, 2008.

[7] Feng Mingjia. Modeling and motion simulation of dual-loop transmission based on Pro / E [J]. Journal of Jilin Agricultural Science and Technology Institute, 2015 (10). 